\title{
CHRISTOPH SORGER
}

\section{La semi-caractéristique d'Euler-Poincaré des faisceaux $\omega$-quadratiques sur un schéma de Cohen-Macaulay}

\author{
Bulletin de la S. M. F., tome 122, no 2 (1994), p. 225-233 \\ <http://www.numdam.org/item?id=BSMF_1994_122_2_225_0>
}

(C) Bulletin de la S. M. F., 1994, tous droits réservés.

L'accès aux archives de la revue «Bulletin de la S. M. F. » (http: //smf.emath.fr/Publications/Bulletin/Presentation.html) implique l'accord avec les conditions générales d'utilisation (http://www.numdam.org/ conditions). Toute utilisation commerciale ou impression systématique est constitutive d'une infraction pénale. Toute copie ou impression de ce fichier doit contenir la présente mention de copyright.

\section{Numdam}




\title{
LA SEMI-CARACTÉRISTIQUE D'EULER-POINCARÉ DES FAISCEAUX $\omega$-QUADRATIQUES SUR UN SCHÉMA DE COHEN-MACAULAY
}

PAR

\author{
Christoph SORGER (*)
}

\begin{abstract}
RÉSUMÉ. - Soit $f: X \rightarrow S$ un morphisme projectif de Cohen-Macaulay de dimension relative pure $n$ entre schémas de type fini sur un corps algébriquement clos. Nous donnons la définition d'un faisceau $\omega_{X / S}$-quadratique (resp. $\omega_{X / S}$-symplectique) de dimension $m$ sur les fibres de $f$ et montrons que le théorème d'invariance mod 2 de Atiyah-Rees, Mumford et Kempf reste valable dans ce cadre plus général. Ensuite, nous l'appliquons à la variété de modules des faisceaux quadratiques semi-stables $\operatorname{Quad}_{X / S}(r)$ de [8] relative à un morphisme de Gorenstein de dimension 1.

Abstract. - Let $f: X \rightarrow S$ be a projective Cohen-Macaulay morphism of pure relative dimension $n$ between schemes of finite type over an algebraically closed field. We give the definition of a $\omega_{X / S}$-quadratic $\left(\omega_{X / S}\right.$-symplectic) sheaf of dimension $m$ on the fibres of $f$ and show that the invariance mod 2 theorem of Atiyah-Rees, Mumford and Kempf is still valid in this more general context. We then apply the theorem to the moduli space of semi-stable quadratic sheaves $\operatorname{Quad}_{X / S}(r)$ of [8] relative to a Gorenstein morphism of dimension 1.
\end{abstract}

\section{Introduction}

Dans ce qui suit, soient $k$ un corps algébriquement clos et $f: X \rightarrow S$ un morphisme projectif de Cohen-Macaulay (voir [EGA IV-2, $\S \S 6.6-6.8]$ ) de dimension relative pure $n$ entre $k$-schémas de type fini.

THÉORÈme 0.1. - Supposons $\operatorname{car}(k) \neq 2$. Soit $\mathcal{F}$ un faisceau cohérent sur $X$ de dimension $m$ sur les fibres de $f$, supposé $\omega_{X / S^{-}}$-quadratique si

(*) Texte reçu le 29 septembre 1992, révisé le 1 février 1993.

C. Sorger, URA 212 du CNRS et UFR de Mathématiques, Université de Paris VII, aile 45-55, $5^{\mathrm{e}}$ étage, 2, place Jussieu F 75251 Paris Cedex 05. email : sorger@mathp7.jussieu.fr.

Classification AMS : 14F 05.

BULLETIN DE LA SOCIÉTÉ MATHÉMATIQUE DE FRANCE 0037-9484/1994/225/\$5.00 (C) Société mathématique de France 
$m \equiv 1 \bmod 4$ et $\omega_{X / S^{-}}$symplectique si $m \equiv 3 \bmod 4$. Alors la fonction $\rho: S \rightarrow \mathbb{N}$, définie par

$$
\rho(s):=\sum_{i \text { pair }} \operatorname{dim} H^{i}\left(X_{s}, \mathcal{F}_{s}\right),
$$

est localement constante modulo 2.

Dans le cas $n=m=1, f$ lisse et $\mathcal{F}$ localement libre, ce théorème - qui remonte à RiEmAnN - est démontré par Atryah [1] et Mumford [6]. Dans le cas $n=m$ quelconque, $f$ lisse et $\mathcal{F}$ localement libre, il est démontré en caractéristique 0 par АтіYAн et ReEs [2], puis en toute caractéristique $\neq 2$ par KEMPF [4] sous l'hypothèse supplémentaire que les fibres de $f$ soient connexes. Le cas $n=2, m=1$ figure dans [8].

L'intérêt de cette généralisation vient du fait qu'il est parfois important de considérer le cas où les fibres de $f$ sont singulières, voire non réduites, non forcément connexes, par exemple si $f: \mathcal{D} \rightarrow \mathcal{C}$ est la courbe universelle de degré $d$ sur une surface projective. Le but de cette note est de montrer comment on peut formuler et démontrer ce théorème dans ce cadre plus général. La démonstration s'inspire de la démonstration récente de KEMPF [4] et est basée sur l'observation plus forte suivante (Proposition 2.1) : si $L^{\bullet}$ est une approximation de longueur $m$ de la cohomologie de $\mathcal{F}$, la structure $\omega$-quadratique (resp. $\omega$-symplectique) donnée par l'isomorphisme symétrique (resp. antisymétrique) $\mathcal{F} \rightarrow \underline{\operatorname{Ext}}_{\mathcal{O}_{X}}^{n-m}\left(\mathcal{F}, \omega_{X / S}\right)$ induit, grâce à la dualité de Serre-Grothendieck, un isomorphisme symétrique (resp. antisymétrique) $L^{\bullet} \rightarrow L^{\bullet *}[-m]$ dans la catégorie dérivée $D_{c}^{b}(S)$.

Je voudrais remercier B. Keller et J. Le Potier pour les discussions que nous avons eues sur le sujet.

Application. - Soit $f: X \rightarrow S$ un morphisme projectif de variétés algébriques irréductibles sur $\mathbb{C}$ et supposons que $f$ soit de Gorenstein de dimension relative 1 . Alors on peut construire, pour $r \in \mathbb{N}$, une variété $Q_{X / S}(r)$ sur $S$ tel que au-dessus de tout point fermé $s \in S$, la fibre s'identifie à l'espace de modules des faisceaux quadratiques de multiplicité $r$ sur $X_{s}$ (cf. [8]). On déduit du théorème que $Q_{X / S}(r)$ a au moins deux composantes.

\section{Notations}

- On note $X_{s}$ la fibre de $f$ au-dessus du point $s$ et si $\mathcal{F}$ est un faisceau sur $X$, on note par $\mathcal{F}_{s}$ la restriction de $\mathcal{F}$ à $X_{s}$.

- Si $\left(L^{\bullet}, d_{L}\right)$ est un complexe de $\mathcal{O}_{S}$-modules, on désigne par $L^{\bullet}[\mathrm{m}]$ le complexe translaté de $m$ places à gauche, de différentielle $(-1)^{m} \mathrm{~d}_{L}$ et

TOME $122-1994-\mathrm{N}^{\circ} 2$ 
par $\tau_{\leq m}\left(L^{\bullet}\right)$ le sous-complexe de $L$ défini par

$$
\cdots \rightarrow L^{m-2} \rightarrow L^{m-1} \rightarrow \operatorname{Ker}\left(\mathrm{d}^{\mathrm{m}}\right) \rightarrow 0 .
$$

- Si $\left(K^{\bullet}, d_{K}\right)$ est un autre complexe de $\mathcal{O}_{S}$-modules, le complexe de $\mathcal{O}_{S}$-modules $\underline{\operatorname{Hom}}^{\bullet}\left(L^{\bullet}, K^{\bullet}\right)$ est défini en degré $n$ par

$$
\prod_{-p+q=n} \underline{\operatorname{Hom}}\left(L^{p}, K^{q}\right),
$$

de différentielle $\mathrm{d}(f)^{p}=d_{K} \circ f^{p}-(-1)^{n} f^{p+1} \circ d_{L}$. Finalement, on note $L^{\bullet *}$ le complexe $\underline{\operatorname{Hom}}\left(L^{\bullet}, \mathcal{O}_{S}\right)$.

- Par $D(S)$, on désigne la catégorie dérivée de $\operatorname{Mod}(S) ; \operatorname{par} D_{c}(S)$ la sous-catégorie pleine des complexes à cohomologie cohérente; par $D^{b}(S)$ la sous-catégorie pleine des complexes bornés à gauche et à droite et enfin, par $D_{c}^{b}(S)$ l'intersection dans $D(S)$ de $D_{c}(S)$ avec $D^{b}(S)$.

\section{Faisceaux $\omega_{X / S}$-quadratiques $\left(\omega_{X / S}\right.$-symplectiques)}

Le cas absolu. - Soit $\left(X, \mathcal{O}_{X}(1)\right)$ un schéma projectif de CohenMacaulay de dimension pure $n$ sur un corps $K$. On note $\omega_{X}$ le faisceau dualisant sur $X$.

Lemme 1.1. - Soit $\mathcal{F}$ un faisceau cohérent de dimension $m$ sur $X$. On $a$ :

(i) $\underline{\operatorname{Ext}}^{i}\left(\mathcal{F}, \omega_{X}\right)=0$ pour $i<n-m$.

(ii) Si $\mathcal{F}$ est de Cohen-Macaulay, alors $\underline{\operatorname{Ext}}^{i}\left(\mathcal{F}, \omega_{X}\right)=0$ pour $i>n-m$.

Démonstration. - En effet, si $(A, \mathcal{M})$ est un anneau local nœthérien de Cohen-Macaulay, $D$ un $A$-module dualisant, alors pour tout $A$-module de type fini $M$ on a la formule (voir [7])

$$
\operatorname{prof}_{A}(M)+\max _{i}\left\{\operatorname{Ext}_{A}^{i}(M, D) \neq 0\right\}=\operatorname{dim}(A),
$$

ce qui démontre la deuxième assertion. La première assertion est bien connue et se déduit par exemple du théorème d'Ischebeck [5] en remarquant que $\operatorname{prof}_{A}(D)=\operatorname{dim}(A)$.

Soit $\mathcal{F}$ un faisceau cohérent de Cohen-Macaulay de dimension $m$ sur $X$. Soit $c=n-m$. On pose :

$$
\mathcal{F}^{\vee}:=\underline{\operatorname{Ext}}_{\mathcal{O}_{X}}^{c}\left(\mathcal{F}, \omega_{X}\right) .
$$

On déduit du lemme que le morphisme canonique d'évaluation $\mathcal{F} \rightarrow \mathcal{F}^{\vee \vee}$ est un isomorphisme. 
DÉfinition 1.2. - On appelle faisceau $\omega_{X}$-quadratique (resp. $\omega_{X}-$ symplectique) sur $X$ la donnée d'un faisceau cohérent de Cohen-Macaulay $\mathcal{F}$ muni d'un isomorphisme $\sigma: \mathcal{F} \rightarrow \mathcal{F}^{\vee}$ satisfaisant à la condition de symétrie $\sigma=\sigma^{\vee}$ (resp. $\left.\sigma=-\sigma^{\vee}\right)$

Considérons le plongement $X \hookrightarrow \mathbb{P}_{K}^{N}$ donné par $\mathcal{O}_{X}(1)$. Soit $d=N-n$. Rappelons qu'on a l'isomorphisme canonique

$$
\underline{\operatorname{Ext}}_{\mathcal{O}_{X}}^{i}\left(\mathcal{F}, \omega_{X}\right) \simeq \underline{\operatorname{Ext}}_{\mathcal{O}_{P}}^{i+d}\left(\mathcal{F}, \omega_{P}\right)
$$

En particulier,

$$
\underline{\operatorname{Ext}}_{\mathcal{O}_{P}}^{i}\left(\mathcal{F}, \omega_{P}\right) \begin{cases}=0 & \text { si } i \neq c+d, \\ \simeq \underline{\operatorname{Ext}}_{\mathcal{O}_{X}}^{c}\left(\mathcal{F}, \omega_{X}\right) & \text { si } i=c+d .\end{cases}
$$

Le cas relatif. - Soit $f: X \rightarrow S$ un morphisme projectif de CohenMacaulay de dimension relative pure $n$ entre $k$-schémas de type fini. On note $\omega_{X / S}$ le faisceau dualisant relatif. Soit $\mathcal{F}$ un faisceau cohérent sur $X$ de dimension $m$ sur les fibres de $f$ et soit $c=n-m$. On pose :

$$
\mathcal{F}^{\vee}:=\underline{\operatorname{Ext}}_{\mathcal{O}_{X}}^{c}\left(\mathcal{F}, \omega_{X / S}\right)
$$

Considérons un plongement de $X$ dans un espace projectif relatif audessus de $S$ :

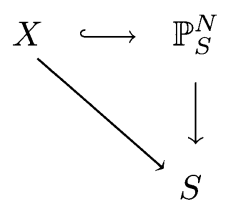

Posons $d=N-n$. Dans la démonstration de la proposition suivante (démontrée dans [8] si $n=m=1$ ), on utilisera la suite spectrale suivante, reliant les groupes de cohomologie $\underline{\operatorname{Ext}}_{\mathcal{O}_{P}}^{i}(\mathcal{F}, \mathcal{G})_{s}$ et $\underline{\operatorname{Ext}}_{\mathcal{O}_{P_{s}}}^{i}\left(\mathcal{F}_{s}, \mathcal{G}_{s}\right)$ pour $s \in S$.

Lemme 1.3. - Soit $p: P \rightarrow S$ un morphisme projectif et lisse, soient $\mathcal{F}$ et $\mathcal{G}$ deux faisceaux cohérents sur $P, S$-plats et $s \in S$. Alors on a la suite spectrale

$$
E_{2}^{p, q}=\underline{\operatorname{Tor}}_{-p}^{\mathcal{O}_{P}}\left(\underline{\operatorname{Ext}}_{\mathcal{O}_{P}}^{q}(\mathcal{F}, \mathcal{G}), \mathcal{O}_{P_{s}}\right) \Longrightarrow \underline{\operatorname{Ext}}_{\mathcal{O}_{P_{s}}^{p+q}}^{p}\left(\mathcal{F}_{s}, \mathcal{G}_{s}\right)
$$

Démonstration. - Soit $\ell$ la dimension relative de $p$. On peut choisir une résolution

$$
0 \rightarrow \mathcal{L}^{-\ell} \rightarrow \cdots \rightarrow \mathcal{L}^{0} \rightarrow \mathcal{F} \rightarrow 0
$$

TOME $122-1994-\mathrm{N}^{\circ} 2$ 
de $\mathcal{F}$ constituée de faisceaux localement libres sur $P$, le morphisme $f$ étant lisse. Considérons le complexe $\mathcal{H}^{\bullet}=\underline{\operatorname{Hom}}^{\bullet}\left(\mathcal{L}^{\bullet}, \mathcal{G}\right)$. La cohomologie de ce complexe s'identifie à $\underline{\operatorname{Ext}}_{\mathcal{O}_{P}}^{i}(\mathcal{F}, \mathcal{G})$ en degré $i$. Soient $\mathcal{K}_{i}=\mathcal{H}^{-i}$ et $F$ le foncteur $? \otimes_{\mathcal{O}_{P}} \mathcal{O}_{P_{s}}$ pour $s \in S$. Puisque $\mathcal{G}$ est $S$-plat, le complexe $\mathcal{K}_{\bullet}$ est $F$-acyclique. Par suite les hypertor $\underline{\text { Tor }}_{i}\left(\mathcal{K}_{\bullet}\right)$ s'identifient à $H_{i}\left(\mathcal{K}_{\bullet} \otimes_{\mathcal{O}_{P}} \mathcal{O}_{P_{s}}\right)$. Considérons la suite spectrale des hypertor :

$$
E_{p, q}^{2}=\underline{\operatorname{Tor}}_{p}^{\mathcal{O}_{P}}\left(H_{q}\left(\mathcal{K}_{\bullet}\right), \mathcal{O}_{P_{s}}\right) \Longrightarrow \underline{\operatorname{Tor}}_{p+q}\left(\mathcal{K}_{\bullet}\right) \text {. }
$$

On pose $E_{2}^{p, q}=E_{-p,-q}^{2}$ et $\mathcal{M}^{i}=\mathcal{K}_{-i}$. La suite spectrale se lit :

$$
E_{2}^{p, q}=\underline{\operatorname{Tor}}_{-p}^{\mathcal{O}_{P}}\left(\underline{\operatorname{Ext}}_{\mathcal{O}_{P}}^{q}(\mathcal{F}, \mathcal{G}), \mathcal{O}_{P_{s}}\right) \Longrightarrow H^{p+q}\left(\mathcal{M}^{\bullet} \otimes_{\mathcal{O}_{P}} \mathcal{O}_{P_{s}}\right) .
$$

La $S$-platitude de $\mathcal{F}$ signifie que $\mathcal{L}_{s}^{\bullet} \rightarrow \mathcal{F}_{s}$ est encore une résolution de $\mathcal{F}_{s}$. On en déduit que $H^{p+q}\left(\mathcal{M}^{\bullet} \otimes_{\mathcal{O}_{P}} \mathcal{O}_{P_{s}}\right)=\underline{\operatorname{Ext}}_{\mathcal{O}_{P_{s}}^{p+q}}^{p}\left(\mathcal{F}_{s}, \mathcal{G}_{s}\right)$, d'où le lemme.

Proposition 1.4. - Supposons que $\mathcal{F}$ est $S$-plat et tel que $\mathcal{F}_{s}$ soit de Cohen-Macaulay pour tout $s \in S$. Alors $\mathcal{F}^{\vee}$ est encore $S$-plat.

Démonstration. - Considérons $\mathcal{F}$ sur $P=\mathbb{P}_{S}^{N}$. Les hypothèses sur $\mathcal{F}$ impliquent qu'il existe une résolution $\mathcal{L} \bullet \rightarrow \mathcal{F}$ par des faisceaux localement libres sur $P$ de longueur $c+d$. On en déduit

$$
\underline{\operatorname{Ext}}_{\mathcal{O}_{P}}^{i}\left(\mathcal{F}, \omega_{P / S}\right)=0 \text { pour } i>c+d .
$$

De la suite spectrale, on déduit que

$$
\underline{\operatorname{Tor}}_{1}^{\mathcal{O}_{P}}\left(\underline{\operatorname{Ext}}_{\mathcal{O}_{P}}^{c+d}\left(\mathcal{F}, \omega_{P / S}\right), \mathcal{O}_{P_{s}}\right)=0
$$

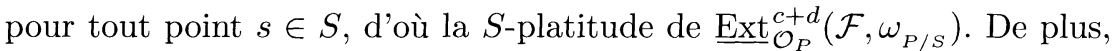
$\underline{\operatorname{Ext}}_{\mathcal{O}_{P}}^{i}\left(\mathcal{F}, \omega_{P / S}\right)=0$ pour $i<c+d$. Montrons maintenant qu'on a l'isomorphisme

$$
\underline{\operatorname{Ext}}_{\mathcal{O}_{X}}^{c}\left(\mathcal{F}, \omega_{X / S}\right) \stackrel{\sim}{\longrightarrow} \underline{\operatorname{Ext}}_{\mathcal{O}_{P}}^{c+d}\left(\mathcal{F}, \omega_{P / S}\right) .
$$

Supposons d'abord $c=0$ et considérons une résolution de $\mathcal{F}$ par des faisceaux localement libres sur $X$ :

$$
\mathcal{L}^{-1} \rightarrow \mathcal{L}^{0} \stackrel{\epsilon}{\longrightarrow} \mathcal{F} \rightarrow 0 .
$$

Soit $\mathcal{E}$ le noyau de $\epsilon$. Alors $\mathcal{E}$ est $S$-plat et $\mathcal{E}_{s}$ est de Cohen-Macaulay pour tout $s \in S$. En particulier, on a $\underline{\operatorname{Ext}}_{\mathcal{O}_{P}}^{i}\left(\mathcal{E}, \omega_{P / S}\right)=0$ pour $i<d$ et le diagramme commutatif de suites exactes :

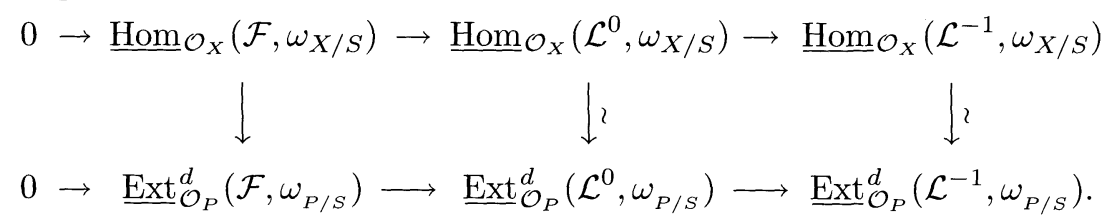

BULLETIN DE LA SOCIÉTÉ MATHÉMATIQUE DE FRANCE 
d'où l'isomorphisme $\underline{\operatorname{Hom}}_{\mathcal{O}_{X}}\left(\mathcal{F}, \omega_{X / S}\right) \stackrel{\sim}{\longrightarrow} \underline{\operatorname{Ext}}_{\mathcal{O}_{P}}^{d}\left(\mathcal{F}, \omega_{P / S}\right)$ par le lemme des cinq. Pour $c>0$, on choisit une résolution de $\mathcal{F}$ par des faisceaux localement libres sur $X$ de longueur $(c-1)$ :

$$
\mathcal{L}^{-c+1} \rightarrow \cdots \rightarrow \mathcal{L}^{0} \stackrel{\epsilon}{\longrightarrow} \mathcal{F} \rightarrow 0 .
$$

Soit $\mathcal{L}^{-c}$ le noyau de la dernière flèche. Alors $\mathcal{L}^{-c}$ est $S$-plat et de plus, de dimension $n$ et de Cohen-Macaulay sur les fibres de $f$. On a un isomorphisme de complexes

$$
\underline{\operatorname{Hom}}_{\mathcal{O}_{X}}^{\mathcal{L}}\left(\mathcal{L}^{\bullet}, \omega_{X / S}\right) \stackrel{\sim}{\longrightarrow} \underline{\operatorname{Ext}}_{\mathcal{O}_{P}}^{\mathrm{d} \bullet}\left(\mathcal{L} \cdot \omega_{X / S}\right)
$$

dont les groupes de cohomologie s'identifient à $\underline{\operatorname{Ext}}_{\mathcal{O}_{X}}^{c}\left(\mathcal{F}, \omega_{X / S}\right)$ et $\underline{\operatorname{Ext}}_{\mathcal{O}_{P}}^{c+d}\left(\mathcal{F}, \omega_{P / S}\right)$ respectivement, d'où l'isomorphisme cherché.

Soit donc $\mathcal{F}$ un faisceau cohérent de dimension $m$ sur les fibres de $f$, $S$-plat et tel que $\mathcal{F}_{s}$ soit de Cohen-Macaulay pour tout $s \in S$. La démonstration du lemme montre que $\operatorname{Ext}_{\mathcal{O}_{X}}^{i}\left(\mathcal{F}, \omega_{X / S}\right)=0$, pour $i \neq c$ et que $\left(\mathcal{F}^{\vee}\right)_{s}=\left(\mathcal{F}_{s}\right)^{\vee}$. Une autre conséquence du lemme est que le morphisme canonique d'évaluation $\mathcal{F} \rightarrow \mathcal{F}^{\vee \vee}$ est un isomorphisme. En effet, soient $\mathcal{K}$ le conoyau et $\mathcal{N}$ le noyau de cette flèche. Pour $s \in S$ on a $\mathcal{K}_{s}=0$, d'où $\mathcal{K}=0$. D'après le lemme précédent, $\mathcal{F}^{\vee \vee}$ est $S$-plat. On sait donc de même que $\mathcal{N}_{s}=0$ pour $s \in S$, d'où $\mathcal{N}=0$.

DÉfInITION 1.5. - On appelle faisceau $\omega_{X / S}$-quadratique (resp. faisceau $\omega_{X / S}$-symplectique) la donnée d'un faisceau cohérent $\mathcal{F}$ sur $X, S$-plat et tel que $\mathcal{F}_{s}$ soit de Cohen-Macaulay pour tout $s \in S$, muni d'un isomorphisme $\sigma: \mathcal{F} \rightarrow \mathcal{F}^{\vee}$ satisfaisant à la condition de symétrie $\sigma=\sigma^{\vee}$ (resp. $\sigma=-\sigma^{\vee}$ )

\section{Démonstration du théorème}

Soit $\mathcal{F}$ un faisceau cohérent sur $X$. On dit qu'un complexe $L \bullet$ formé de $\mathcal{O}_{S}$-modules cohérents localement libres est une approximation de la cohomologie de $\mathcal{F}$ si pour tout changement de base $S^{\prime} \stackrel{g}{\longrightarrow} S$,

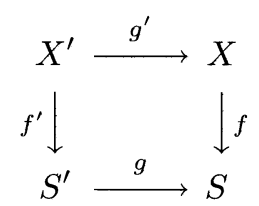

on a $H^{i}\left(g^{*} L^{\bullet}\right)=\mathrm{R}^{i} f_{*}^{\prime} g^{*} \mathcal{F}$. En particulier, pour un point $s \in S$, on a $H^{i}\left(L_{s}^{\bullet}\right)=H^{i}\left(X_{s}, \mathcal{F}_{s}\right)$. On dit que l'approximation $L^{\bullet}$ est de longueur $m$ si $L^{i}=0$ pour $i<0$ et $i>m$.

TOME $122-1994-\mathrm{N}^{\circ} 2$ 
Proposition 2.1. - Soit $(\mathcal{F}, \sigma)$ un faisceau $\omega_{X / S}$-quadratique (resp. $\omega_{X / S}$-symplectique) de dimension $m$ sur les fibres. Alors il existe une approximation $L^{\bullet}$ de la cohomologie de $\mathcal{F}$ de longueur $m$ munie d'un isomorphisme symétrique (resp. antisymétrique) dans $D_{c}^{b}(S)$

$$
\psi: L^{\bullet} \longrightarrow L^{\bullet *}[-m]
$$

induit par $\sigma$.

Démonstration. - GROTHENDIECK démontre l'existence d'une approximation finie pour un morphisme propre en utilisant la cohomologie de Čech. Dans le cas d'un morphisme projectif de Cohen-Macaulay on peut procéder de la manière suivante. Soit

$$
0 \rightarrow \mathcal{L}^{-n} \rightarrow \cdots \rightarrow \mathcal{L}^{0} \rightarrow \mathcal{F} \rightarrow 0
$$

une résolution de $\mathcal{F}$ par des faisceaux cohérents tels que $\mathcal{L}^{i}$ soit localement libre et tels que $\mathrm{R}^{q} f_{*} \mathcal{L}^{i}=0, q \neq n$ pour $i=0, \ldots,-n+1$. Remarquons que le noyau $\mathcal{L}^{-n}$ de la dernière flèche satisfait de même à $\mathrm{R}^{q} f_{*} \mathcal{L}^{-n}=0$, $q \neq n$. Soient $K^{\bullet}=\mathrm{R}^{n} f_{*}\left(\mathcal{L}^{\bullet}\right)[-n]$ et $L^{\bullet}=\tau_{\leq m}\left(K^{\bullet}\right)$. Dans $D(S)$, l'inclusion de $L^{\bullet}$ dans $K^{\bullet}$ est un isomorphisme, les $\mathrm{R}^{q} f_{*}(\mathcal{F})$ étant nul pour $q>m$. Par le théorème de changement de base, les $L^{i}$ sont localement libres et commutent aux changements de base. Ainsi $\mathcal{L}^{\bullet}$ est une approximation de longueur $m$ de la cohomologie de $\mathcal{F}$.

Considérons maintenant, dans $D_{c}(S)$, le diagramme d'isomorphismes suivant :

$$
\begin{aligned}
& L^{\bullet} \simeq \mathrm{R} f_{*}(\mathcal{F})
\end{aligned}
$$

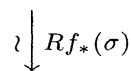

$$
\begin{aligned}
& \mathrm{R} f_{*}\left(\mathcal{F}^{\vee}\right) \simeq \mathrm{R} f_{*} \operatorname{R} \underline{\operatorname{Hom}}\left(\mathcal{F}, \omega_{X / S}\right)[c] \\
& \left(\operatorname{car} \underline{\operatorname{Ext}}^{i}\left(\mathcal{F}, \omega_{X / S}\right)=0, i \neq c\right) \\
& \simeq \underline{\mathrm{Rom}}^{\bullet}\left(\mathrm{R} f_{*}(\mathcal{F}), \mathcal{O}_{S}\right)[c-n] \\
& \text { (dualité de Grothendieck [3]) } \\
& \simeq \mathrm{R}^{\operatorname{Hom}} \cdot\left(L^{\bullet}, \mathcal{O}_{S}\right)[-m] \\
& \simeq L^{* *}[-m] \text {. }
\end{aligned}
$$

Par composition, on déduit l'isomorphisme $\psi: L^{\bullet} \stackrel{\sim}{\rightarrow} L^{\bullet *}[-m]$ dans $D_{c}^{b}(S)$. L'assertion concernant la symétrie de $\psi$ est bien sûr une conséquence de la symétrie de $\sigma$. 
Corollaire 2.2. - Supposons que $\operatorname{car}(k) \neq 2$. Sous les hypothèses de la proposition, on peut trouver, localement, une approximation $Z \cdot$ de longueur $m$ formée de faisceaux cohérents libres et munie d'un isomorphisme symétrique (resp. antisymétrique) de complexes (et non seulement un isomorphisme dans $\left.D_{c}^{b}(S)\right)$

$$
\Sigma: Z^{\bullet} \longrightarrow Z^{\bullet *}[-m]
$$

induit par $\sigma$.

Démonstration. - La question étant locale, on peut supposer que $S=$ $\operatorname{Spec}(A)$, où $A$ est un anneau nœthérien. Comme $L^{\bullet}$ est formé de faisceaux cohérents localement libres, et comme $\operatorname{Coh}(S)$ a suffisamment d'objets projectifs, $\psi$ est un isomorphisme dans la catégorie homotopique $K_{c}^{b}(S)$. Soit $\chi$ un relèvement de $\psi$ dans $C_{c}^{b}(S)$. Pour $i=0, \ldots, m$, on pose

$$
\varphi^{i}=\frac{1}{2}\left(\chi^{i}+{ }^{t} \chi^{m-i}\right) \quad \text { ou } \quad \varphi^{i}=\frac{1}{2}\left(\chi^{i}-{ }^{t} \chi^{m-i}\right)
$$

selon que $(\mathcal{F}, \sigma)$ est $\omega_{X / S}$-quadratique ou $\omega_{X / S}$-symplectique. Remarquons que ${ }^{t} \varphi^{i}=\varphi^{m-i}$ dans le cas quadratique, ${ }^{t} \varphi^{i}=-\varphi^{m-i}$ dans le cas symplectique et que $\varphi$ est un quasi-isomorphisme.

Fixons $s \in S$. Il existe, au voisinage de $s$, un complexe $Z^{\bullet}$ de longueur $m$, formé de faisceaux cohérents libres et tel que $d_{\mid s}=0$, et un quasi-isomorphisme $u: Z^{\bullet} \rightarrow L^{\bullet}$. Considérons $\Sigma: Z^{\bullet} \rightarrow Z^{\bullet *}[-m]$ défini par $\Sigma={ }^{t} u[-m] \circ \varphi \circ u$. Alors $\Sigma$ est symétrique (resp. antisymétrique) et $\Sigma_{\mid s}$ est un isomorphisme. Au voisinage de $s, \Sigma$ restera un isomorphisme, d'où le corollaire.

Montrons comment le corollaire entraîne le théorème. On reprend l'argument de KEMPF [4]. Soient $m=2 k+1$ et $(\mathcal{F}, \sigma)$ un faisceau $\omega_{X / S}$-quadratique (resp. $\omega_{X / S}$-symplectique) si $m \equiv 1(\bmod 4)(\operatorname{resp}$. $m \equiv 1(\bmod 4))$. Considérons l'isomorphisme $\Sigma$ :

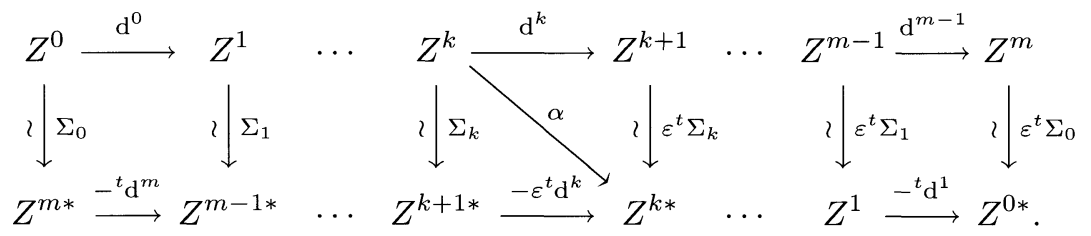

où l'on a $\varepsilon=(-1)^{k}$. On voit qu'on a

$$
\rho(s) \bmod 2=\sum_{i \text { pair }} \operatorname{dim} Z^{i}-\operatorname{rang} \alpha \bmod 2 .
$$

TOME $122-1994-\mathrm{N}^{\circ} 2$ 
Le morphisme $\alpha$ est antisymétrique et par suite de rang pair ce qui démontre le théorème.

\section{BIBLIOGRAPHIE}

[1] Aтiyah (M.F.). - Riemann Surfaces and Spin Structures, Ann. Scient. Éc. Norm. Sup., $4^{\text {e }}$ série, t. 4, 1971, p. 47-62.

[2] M. F. Atiyah (M.F.) and Rees (E.). - Vector bundles on Projective 3-space, Invent. Math., t. 35, 1976, p. 131-153.

[3] Hartshorne (R.). - Residues and Duality. - Lecture Notes in Math. 20, Springer-Verlag, Berlin, 1966.

[4] Kempf (G.). - Deformations of Semi-Euler Characteristics, Amer. J. of Math., t. 114, 1992, p. 973-978.

[5] Matsumura (H.). - Commutative Ring Theory. - Cambridge Studies in Advanced Math. 8, Cambridge University Press, Cambridge, 1980 .

[6] Mumford (D.). - Theta-Characteristics of an Algebraic Curve, Ann. Scient. Éc. Norm. Sup., $4^{\text {e }}$ série, t. 4, 1971, p. 181-192.

[7] Peskine (C.). - Introduction algébrique à la géométrie projective. - Cours de DEA, Univ. Paris VI, 1990.

[8] Sorger (C.). - Thêta-caractéristiques des courbes tracées sur une surface lisse, J. reine angew. Math. (Journal de Crelle), t. 435, 1993, p. 83-118. 